\title{
Preface: syntactic and composite foams special section
}

\author{
G. M. Gladysz $\cdot$ K. K. Chawla $\cdot$ A. R. Boccaccini
}

Received: 11 April 2012/ Accepted: 12 April 2012/Published online: 1 May 2012

(C) Springer Science+Business Media, LLC 2012

A conference titled "Syntactic and Composite Foams III" (SCF III), under the auspices of Engineering Conferences International (ECI), was held in May/June 2011 in Cetraro (Calabria), Italy. This conference was the third in the series. The conference was chaired by Dr. G. M. Gladysz (Trelleborg Offshore US), Professor K. K. Chawla (University of Alabama at Birmingham, Birmingham, AL, USA), and Professor A. R. Boccaccini (University of Erlangen-Nuremberg). An international organizing committee with representatives from universities, industries, and government laboratories was involved in reviewing the papers for publication in this special section of Journal of Materials Science.

The first, SCF I, was held in Banff, Canada, in 2004. Papers from that conference were published in a Special Issue on Syntactic and Composite Foams in the Journal of Materials Science (Volume 41, Number 13). The second, SCF II, was held in Davos, Switzerland, in 2007 and the papers were again published in a special issue of the Journal of Materials Science (Volume 44, Number 6). These first two conferences, also held under the auspices of ECI, provided an excellent forum for discourse among scientists and engineers from the academia, industry, and research laboratories on the subject of syntactic and composite foams.

G. M. Gladysz ( $\square)$

Trelleborg Offshore, Mansfield, MA, USA

e-mail: gary.gladysz@trelleborg.com

K. K. Chawla

University of Alabama, Birmingham, AL, USA

A. R. Boccaccini

University of Erlangen, Erlangen, Germany
Composite foams are cellular materials consisting of a binder phase, reinforcing/functional phase(s), and voids distributed throughout. The reinforcing phase can be solid or hollow. Syntactic foams are a particular type of composite foam. In this case the reinforcing particles are hollow microspheres also called microballoons with a polymer, metal, or ceramic binder material. Analogous to the fibrous composites in many ways, composite foams are multifunctional materials which offer many ways to tailor a wide range of physical and mechanical properties and functionalities. The fact that they are foams, i.e., exhibiting a designed void structure, suggests that they are suited for specific applications such as those requiring low density, high permeability and/or bio-scaffolding. The incorporation of functional phases (fibers or particles) or microballoon shell material can allow one to tailor the structure to property: mechanical, thermal, electrical etc. In syntactic foams, the presence of hollow microspheres results in a foam of low density, high compressive strength, and generally a low thermal expansion coefficient. These foams are typically used in applications that take advantage of their very high specific properties, pore structure, and energy absorbing characteristics. If a reinforcing and/or functional phase is added to a foam, we get a composite foam with additional desirable characteristics. These additions expand the potential applications beyond those of the traditional deep sea buoyancy into more complex structures for use in aerospace, automotive, communications, biomedical, electronics, sporting, and transportation industries.

We now have held the third in the series, SCF III and are publishing the third JMS Special issue containing peerreviewed papers based on research presented at the conference. We started planning of SCF I in 2002 while this batch has a target publication date of 2012, which allows us to take a look back over the last decade and also project 


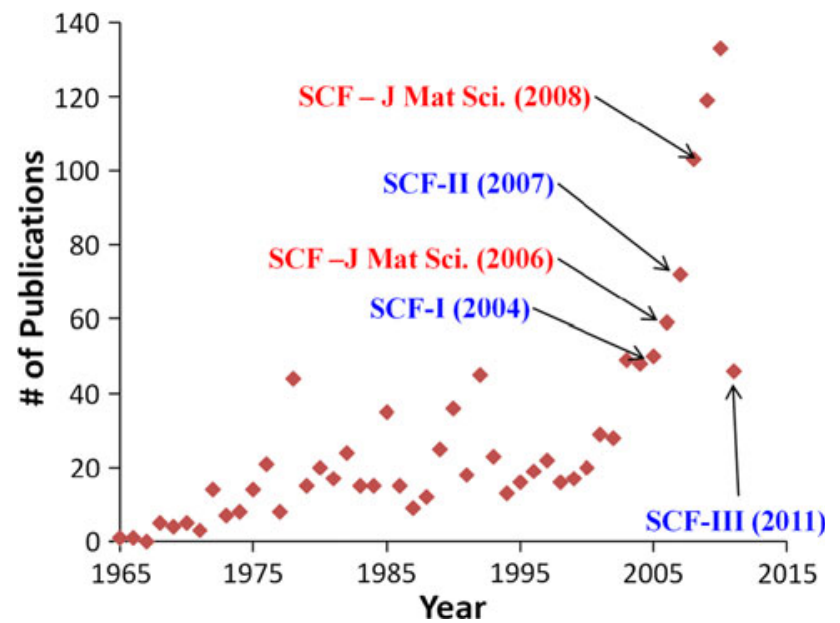

Fig. 1 Increase in syntactic and composite foams publications from 1965 to May 2011. Note the dramatic upturn starting in 2003 and continues today. The graph also includes the years this conference was held (in red) and the year the Special Issue published in the Journal of Materials Science was published (Color figure online)

what the future might hold for this very interesting class of materials. For a look back, we have done a key word search in ScienceDirect on key terms which are important descriptors in identifying syntactic and composite foams.

Figure 1 shows the result of this search and details the number of publications each year from 1965, the year of the first syntactic/composite foam publication, through May 2011 the date SCF III was held. Also included on the graph are the years that the SCF conferences were held, in blue and the years the special issues were published in this journal, in red. The trend is apparent and not much explanation is need. Since 2003/2004 there has been an exponential growth in the number of publications. One can conclude from these data that the overall interest in this class of materials is growing, possibly because of the unique and tailorable properties they exhibit; driven by a need for increasing functionality; whether for reduced weight for fuel saving or for optimum functionality of biocompatible structures.

Looking back over the three conferences, there are a few distinct categories or overarching themes into which the presentations and papers fall, namely:

1. Biomaterials,

2. Thermal management,

3. Low density structural applications including buoyancy and aerospace,

4. Fundamental characterization and modeling, and

\section{Energy absorption}

These are the main applications for this class of material and most talks can be neatly categorized into these areas. Indeed over the years, researchers have built on these foundations to develop and investigate applications and structures with increasing complexity. There are some that involve entirely new applications like the use hollow coated glass microballoons for waste water treatment. Other topics included:

1. Hollow structures for whispering gallery mode resonator-based mechanical sensors for monitoring the structural health of components.

2. Plug-assist materials for thermoforming processes

3. Incorporation of additional phases, carbon nanofibers, nanotubes

4. Applications involving sporting goods

The SCF III brought scientists and engineers from ten countries together to discuss the latest research in the field of high performance foams. As in the other conferences there was a very broad range of talks that ranged from high temperature aerospace heat-shield applications for re-entry vehicles, to deep sea and biomedical implant applications.

The conference concluded with round table discussions on the broad range of topics with the goal to build partnerships between research and development, manufacturers, and users. Some of the topics included:

- Bio applications

- Deep sea applications

- Testing/characterization techniques

- Processing and performance

- Emerging applications

- Emerging materials

The move in industry is for increasing functionality; whether for reduced weight for fuel saving or for optimum functionality of biocompatible structures.

Acknowledgements We acknowledge the generous support, financial and otherwise, of the following organizations and people: Engineering Conferences International; we are especially grateful to Ms. Barbara Hickernell, Mr. Kevin Korpics, and Ms. Arlene Conway of ECI for their help. We also thank the ECI liaison Dr. Ram Darolia. Thanks go to Trelleborg Offshore for again hosting the Meet \& Greet. We like to thank the Journal of Materials Science, especially editor M. Grant Norton and Editor-in-Chief C. Barry Carter for agreeing on the publication of this special section. Last but not least, we are grateful to all the participants for making this event a great success. All three of us are looking forward to SCF IV! 